\title{
A One-Pot Route to Fine-Tuned Hypercrosslinked Polymer Solid Acid Catalysts
}

Alexander Blocher, ${ }^{\text {a+ }}$ Florian Mayer, ${ }^{a+}$ Paul Schweng, ${ }^{a}$ Teresa M. Tikovits, ${ }^{\text {a }}$ Robert T. Woodward ${ }^{\text {a* }}$ alnstitute of Materials Chemistry and Research, Faculty of Chemistry, University of Vienna, Währinger Straße 42, 1090 Vienna, Austria.

'These authors contributed equally to this work.

*email: robert.woodward@univie.ac.at

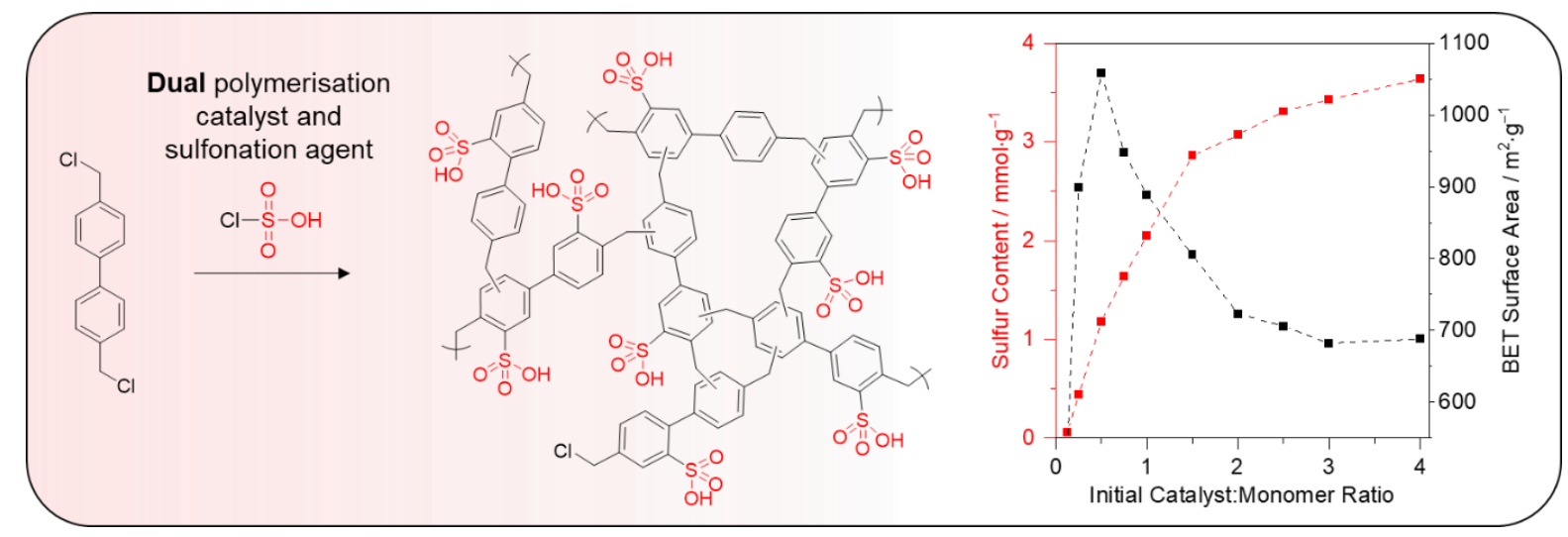

\section{Abstract}

Highly acidic sulfonated polymers are promising heterogeneous catalysts due to their excellent chemical and thermal stability, as well as the possibility of employing low-cost synthesis routes. However, their production is time consuming and control of their chemical and physical properties is often difficult. Here, we produce sulfonated hypercrosslinked polymers using chlorosulfonic acid as a dual polymerisation catalyst and sulfonation agent, consolidating a conventional two-step process into a one-pot reaction. The synthesis time of the networks is reduced from 5-6 days to $<24$ hours, while significantly lowering or eliminating the requirement of some reagents. Furthermore, these sulfonated networks display superior acid site densities and porous properties to conventional equivalents. By varying the ratio of aromatic monomer with the multifunctional chlorosulfonic acid at the synthetic stage, excellent control over the polymers' porous properties and sulfonation density is achieved, permitting judicious catalyst design. The ability of these polymers to act as heterogeneous acid catalysts is demonstrated via the hydrolysis of cyclohexyl acetate. Therein, it is shown that fine-tuning polymers has a dramatic effect on their catalytic performance and allows for the identification of key catalyst properties for optimal conversion. 


\section{Introduction}

An increased demand for more sustainable approaches in the chemical industry is being driven by considerable socio-economic and environmental changes. As a result, reusable, energy efficient, and selective catalysts have become a key pillar in the principles of green chemistry. ${ }^{1}$ By the immobilisation of homogeneous catalysts or equivalent chemical functionality onto inert insoluble frameworks, effective heterogeneous catalysts that adhere to these principles can be realised. ${ }^{2}$ Heterogeneous catalysts offer advantages over homogeneous equivalents, such as use in continuous flow processes, simple separation and recovery from reactants and products, and straightforward recycling. However, reduced catalytic activity, high-costs, and poor stabilities can often hold-back the application of such catalysts in industry. ${ }^{3}$ Immobilisation requires the synthesis of pre-functionalised frameworks, followed by the anchoring of active sites, often requiring multistep procedures and expensive reagents. ${ }^{4}$ Moreover, immobilisation can alter the microenvironment of active sites, leading to decreased activity or accessibility for reactants. To make heterogeneous catalysts competitive with homogeneous equivalents, their active sites need to be dense and accessible, their production should exploit low-cost design, and their implementation has to offer simple operation and compatibility with many facets of modern catalysis.

Porous organic polymers (POPs) are excellent candidates for heterogeneous catalysis due to their high surface areas, tuneable textural properties, and excellent chemical, thermal, and mechanical stabilities. The vast number of synthetic routes to POPs also permit readily modifiable chemical functionality and/or broad bottom-up design. Common examples of POPs include covalent organic frameworks (COFs) ${ }^{5}$ and conjugated microporous polymers (CMPs), ${ }^{6}$ both of which have shown great potential in separation and catalysis applications. However, many POP synthesis routes require precious metal catalysts, which are typically not recycled, significantly driving up cost and hindering scale-up. Furthermore, specifically polymerisable groups in monomeric material are often required for the formation of POP networks. Such monomers are seldomly commercially available or are expensive.

Hypercrosslinked polymers (HCPs) are a low-cost class of POPs with excellent tunability. ${ }^{7}$ HCPs are densely crosslinked amorphous networks, produced using simple Friedel-Crafts chemistry. One approach to HCPs utilises non-functional aromatic compounds (i.e. without specifically polymerisable groups) that are 'knitted' together in a variety of ways, such as using external crosslinkers, ${ }^{8}$ solvent stitching reactions, ${ }^{9}$ or Scholl reactions. ${ }^{10,11}$ Another approach produces HCPs via self-condensation reactions of chloromethyl ${ }^{12}$ or methyl ether ${ }^{13}$ moieties with aromatic 
carbon to form crosslinks. Hypercrosslinking requires only abundant Fe- or Al-based Lewis acid catalysts ${ }^{14}$ or simple organic acid catalysts. ${ }^{15,16}$ Owing to their low costs and broad design scope, HCPs are developed for a variety of applications including gas separation and storage, ${ }^{17}$ solid state extraction, ${ }^{18}$ and energy storage. ${ }^{19}$ HCPs also show promise in heterogeneous catalysis, both as inert frameworks or as active materials. ${ }^{20} \mathrm{HCP}$-based catalysts were employed for a wide array of transformations, ranging from biomass conversion ${ }^{21,22}$ to the photocatalytic reduction of $\mathrm{CO}_{2}{ }^{23,24}$ High surface areas allow for an abundance of catalytically active sites and hierarchically porous structures are beneficial to the mass transfer of reactants and products. As such, HCPs show excellent ability in catalysis and are ideal candidates for industrial applications in accordance with the principles of green chemistry.

Through treatment of HCPs with sulfonating agents, heterogeneous acid catalysts combining the desirable properties of HCPs with high acid-site densities, so called sulfonated HCPs (SHCPs), are produced. Dong et al. synthesised sulfonated hypercrosslinked benzene networks containing up to $2 \mathrm{mmol} \cdot \mathrm{g}^{-1}$ of $\mathrm{SO}_{3} \mathrm{H}$ for the selective dehydration of $\mathrm{D}-(-)$-fructose to 5 -hydromethylfurfual, reaching conversions of $>90 \% .{ }^{21}$ An analogous set of materials catalysed the selective conversion of carbohydrate substrates to ethyl levulinate in yields of up to $67 \% .^{22}$ Therein, it was speculated that multiple variables, including surface area, catalyst dose, and acid density played a critical role in the efficiency of the catalytic transformation. Sulfonated carbazole-based HCPs with impressive $\mathrm{SO}_{3} \mathrm{H}$ densities of $3.7 \mathrm{mmol} \cdot \mathrm{g}^{-1}$ were developed for the esterification of a number of fatty acids to produce biofuels, reaching conversions of up to $99 \% .{ }^{25}$ Elsewhere, a SHCP containing a phosphonium salt in its structure was used for the two-phase hydrolysis of cyclohexyl acetate to cyclohexanol, achieving conversions and selectivity of up to 93 and $95 \%$, respectively. ${ }^{26}$ SHCPs have also shown promise as selective adsorbents, including for propene/propane separation, ${ }^{27,28}$ heavy metal adsorption, ${ }^{29}$ and water pollutants. ${ }^{30}$

In conventional approaches, sulfonic acid groups are introduced via the post-synthetic treatment of HCPs using sulfonating agents, commonly chlorosulfonic acid. This route involves the swelling of the HCP network in inert solvent, followed by treatment with large excesses of the sulfonating agent. Although effective, control over the degree of sulfonation and the resulting porous properties of the catalyst is lacking. Furthermore, the approach is cumbersome, requiring the complete synthesis and work up of the HCP prior to sulfonation, resulting in a synthesis time of up to 6 days.

We present a strategy for the synthesis of SHCPs in a one-pot approach, significantly reducing reaction times and reagent use compared to conventional approaches. These SHCPs allow for 
careful catalyst design and have superior properties to SHCPs obtained using other synthetic procedures. Hazardous organic solvents and reagents are significantly reduced or eliminated altogether, making the method simple, safer, cleaner, and more economical. To assess the potential of these SHCPs as solid acid catalysts, we report their application to the hydrolysis of cyclohexyl acetate. The properties of these finely-tuned SHCPs had a dramatic effect on their performance in this two-phase reaction, allowing for the identification of key catalyst properties for optimal catalytic performance.

\section{Results and Discussion}

We synthesised ten SHCPs from 4,4'-bis(chloromethyl)biphenyl using chlorosulfonic acid as a dual polymerisation catalyst and sulfonation agent (a general reaction scheme is shown in Figure 1a). Throughout the set, the ratio of chlorosulfonic acid to the aromatic monomer was varied to investigate the effect on the resulting SHCPs' textural and chemical properties. The progression from SHCP-1 to SHCP-10 follows an increasing catalyst-to-monomer ratio, precise values of which are given in Table 1. Briefly, 4,4'-bis(chloromethyl)biphenyl was dissolved in 1,2dichloroethane (1,2-DCE) and cooled using an ice bath, after which a solution of chlorosulfonic acid in 1,2-DCE was added to initiate polymerisation. Upon formation of a solid, the mixture was sealed and heated at $80{ }^{\circ} \mathrm{C}$ for up to $22 \mathrm{~h}$. The resulting solid was then washed with methanol before drying to yield the SHCP. More details are given in the supporting information. It is worth noting that a shortened procedure in which the heating time was reduced to $3 \mathrm{~h}$ showed no apparent detriment to resulting SHCP properties. Polymers were typically produced in yields of $>90 \%$ when considering the incorporation of the acid catalyst as a sulfonic acid group. Networks ranged from a beige to dark brown colour with increasing catalyst to monomer ratios (Figure S1). All SHCPs were produced at least four times to ensure reproducibility.

We confirmed the successful formation of SHCPs using ${ }^{13} \mathrm{C}$ cross-polarisation/magic angle spinning solid-state NMR (CP/MAS ssNMR) (Figure 1b). Signals at $\sim 38 \mathrm{ppm}$ are assigned to methylene bridges from newly formed crosslinks in all cases. A weak signal assigned to $\mathrm{C}-\mathrm{Cl}$ at $\sim 42 \mathrm{ppm}$ can be seen in SHCP-1 and decreases in intensity through to SHCP-3, indicative of incomplete crosslinking due to low concentrations of chlorosulfonic acid. A signal at $\sim 120 \mathrm{ppm}$ is found in the shoulder of a larger peak in samples SHCP-5 - SHCP-10, assigned to the C-S bond formed during sulfonation. At lower ratios of catalyst to monomer this peak can no longer be seen due to reduced degrees of sulfonation. All SHCPs showed strong signals at $\sim 129$ and $\sim 139 \mathrm{ppm}$, corresponding to aromatic $\left(\mathrm{C}_{\mathrm{Ar}}-\mathrm{H}\right)$ and quaternary, or substituted, aromatic carbons $\left(\mathrm{C}_{\mathrm{Ar}}-\mathrm{R}\right)$, respectively. Although difficult to quantify due to overlapping peaks, it is clear that the ratio 
between substituted and unsubstituted aromatic peaks changes across the polymer set. Increasing amounts of chlorosulfonic acid in the initial formulation appear to increase the ratio of substituted to unsubstituted aromatic carbon. Considering the increase in C-S peak as well as the disappearance of the $\mathrm{C}-\mathrm{Cl}$ peak with higher initial concentrations of chlorosulfonic acid, this is in line with expectation. We also confirmed successful polymer formation and sulfonation using Fourier-transform infrared spectroscopy (Figure S2). There, the presence of bands assigned to both $\mathrm{SO}_{3} \mathrm{H}$ and $\mathrm{C}-\mathrm{S}$ bonds emerged with increasing ratios of catalyst to monomer, confirming increasing sulfonation. The diminishing of the $\mathrm{C}-\mathrm{Cl}$ band across the series was also observed, in good agreement with ssNMR.

a

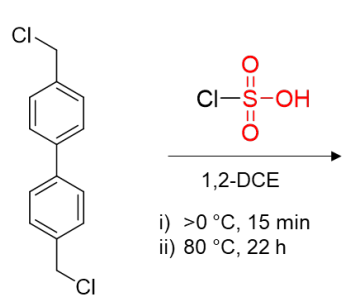

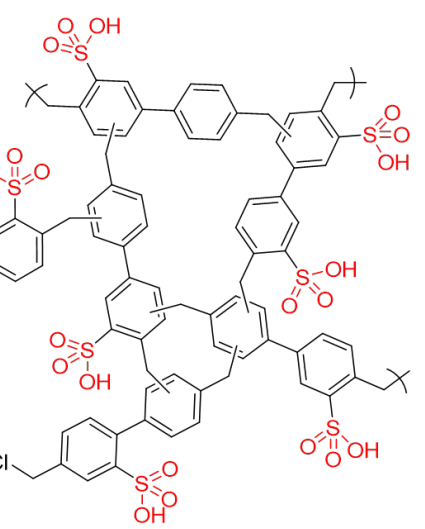
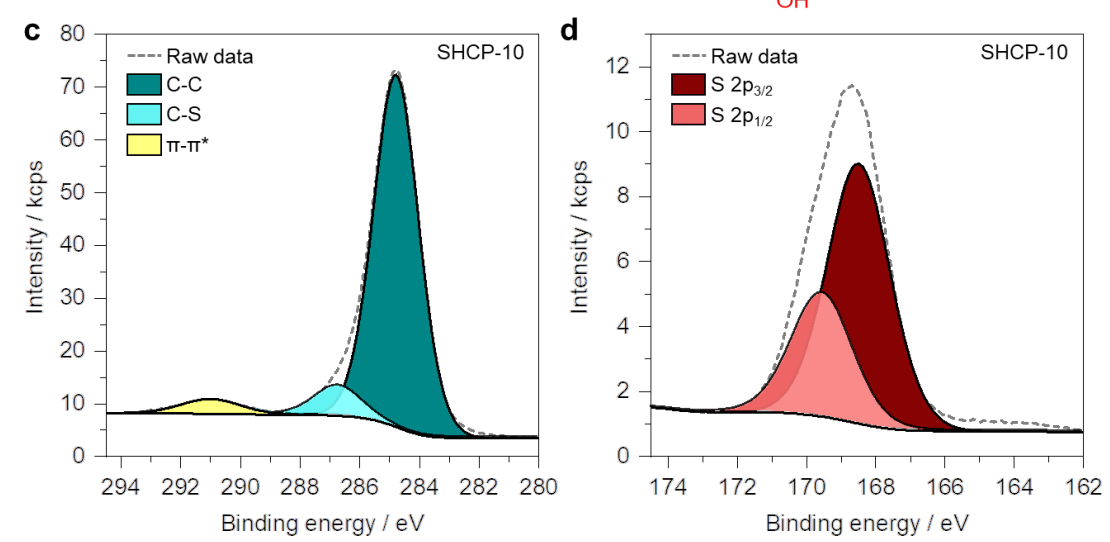

b

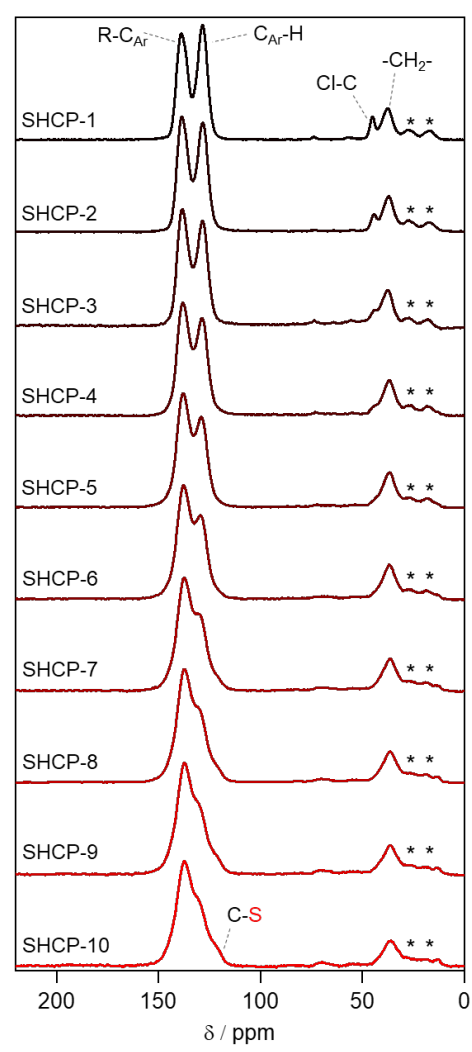

Figure 1. Sulfonated hypercrosslinked polymer characterisation. a) Reaction scheme for the one-pot synthesis of SHCPs. b) ${ }^{13} \mathrm{C}$ CP/MAS solid state NMR spectra for all SHCPs, with increasing catalyst to monomer ratio used in the production of SHCP-1 - SHCP-10. * represents spinning side bands. c) X-ray photoelectron C 1s spectrum for SHCP10. d) X-ray photoelectron S 2 p spectrum for SHCP-10. (grey dotted lines in c) and d) represent raw data).

We used X-ray photoelectron spectroscopy (XPS) to gain a more in-depth understanding of SHCPs' chemical compositions (XPS derived quantitative data for all polymers is provided in Table S1). The main component of the high-resolution $C 1$ s spectra observed at a binding energy of $284.8 \mathrm{eV}$ is attributed to $\mathrm{C}-\mathrm{C}$ bonding, encompassing both sp2 aromatic carbon and sp3 carbon 
in methylene crosslinks (SHCP-10 shown in Figure 1c). A peak of lower intensity is observed at $286.8 \mathrm{eV}$, corresponding to C-S. A broad, low intensity $\pi-\pi^{*}$ shake-up feature is also observed at $291 \mathrm{eV}$. High resolution S 2p spectra showed a typical asymmetrical peak for a sulfonic acid moiety (SHCP-10 shown in Figure 1d). The peaks at binding energies of $168.5 \mathrm{eV}$ and $169.5 \mathrm{eV}$ are assigned to $S 2 p_{3 / 2}$ and $2 p_{1 / 2}$, respectively. ${ }^{31}$ This peak is present throughout all sulfonated networks, confirming the vast majority of the sulfur present exists in sulfonic acid groups. Further evidence for this is provided by a consistent ratio of $1: 3$ for $S$ to $O$ across all samples, as would be expected for sulfonic acid groups. Increasing catalyst to monomer ratios led to higher concentrations of sulfonic acid sites in the networks, reaching a maximum sulfur content of $4.6 \mathrm{mmol} \cdot \mathrm{g}^{-1}$ in SHCP-10, as determined by XPS. This is amongst the highest degrees of sulfonation reported for hypercrosslinked polymers, with another similarly high example reported for carbazole based networks $\left(3.7 \mathrm{mmol} / \mathrm{g}\right.$, as determined by elemental analysis). ${ }^{25} \mathrm{We}$ hypothesize that polymerisation occurs more rapidly than sulfonation, leading to the uniform distribution of chlorosulfonic acid throughout the HCP structure. This dispersion facilitates excellent acid densities throughout networks upon sulfonation, as it eliminates the need for the sulfonation agent to permeate into a pre-formed structure.

We employed CHNS-O elemental analysis (EA) to measure the bulk chemical composition of the networks (Table S2). The EA results showed a similar trend to XPS, with sulfonation densities increasing upon an increase in catalyst-to-monomer ratio, confirming a good dispersion of acidsites both at the surface and throughout the bulk of the networks. At higher catalyst-to-monomer ratios $(\geq 2)$, the EA results deviate quite significantly from XPS, with SHCP-10 measured as containing $3.8 \mathrm{mmol} \cdot \mathrm{g}^{-1}$ of $\mathrm{SO}_{3} \mathrm{H}$ compared to $4.6 \mathrm{mmol} \cdot \mathrm{g}^{-1}$ in XPS. This is due to water adsorption owing to the increased hydrophilicity of the networks with higher sulfonation densities. This can be observed in the $\mathrm{S}: \mathrm{O}$ atomic ratios calculated from EA, which ideally should be $1: 3$ but is as high as 1:5.4 in SHCP-10. Furthermore, XPS cannot consider $\mathrm{H}$ when determining at.\% of elements present, leading to a slight overestimation. Therefore, the real concentration of $\mathrm{SO}_{3} \mathrm{H}$ likely lies somewhere between the values determined by XPS and EA.

We measured $\mathrm{N}_{2}$ sorption isotherms for all SHCP polymers at $77 \mathrm{~K}$ to gain information about their porous properties (Figure 2a and S3). The sulfonated networks showed excellent reproducibility regarding their porous properties. Networks displayed characteristics of both Type I and Type IVa isotherms, with uptake at low relative pressures signalling microporosity and significant hysteresis observed upon desorption deriving from capillary condensation, indicative here of mesoporosity/macroporosity. All SHCPs displayed $\mathrm{H} 2$ type hysteresis curves, indicative of broad 
pore size distributions with narrow pore necks. ${ }^{32}$ The absence of a closed hysteresis loop is common in the isotherms of HCPs and likely due to the swelling of the networks during adsorption. ${ }^{33}$ With increasing sulfonation density the hysteresis loops upon desorption appear less pronounced, indicating that the mesopore component of the networks decreases. This is

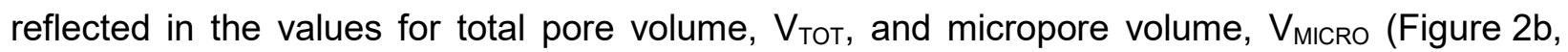
Table 1), which confirmed the broad pore size distribution. Intriguingly, the micropore volume remains relatively constant with increasing degrees of sulfonation from SHCP-3 onward, permitting the careful control of micropore-to-mesopore/macropore ratio in SHCPs.
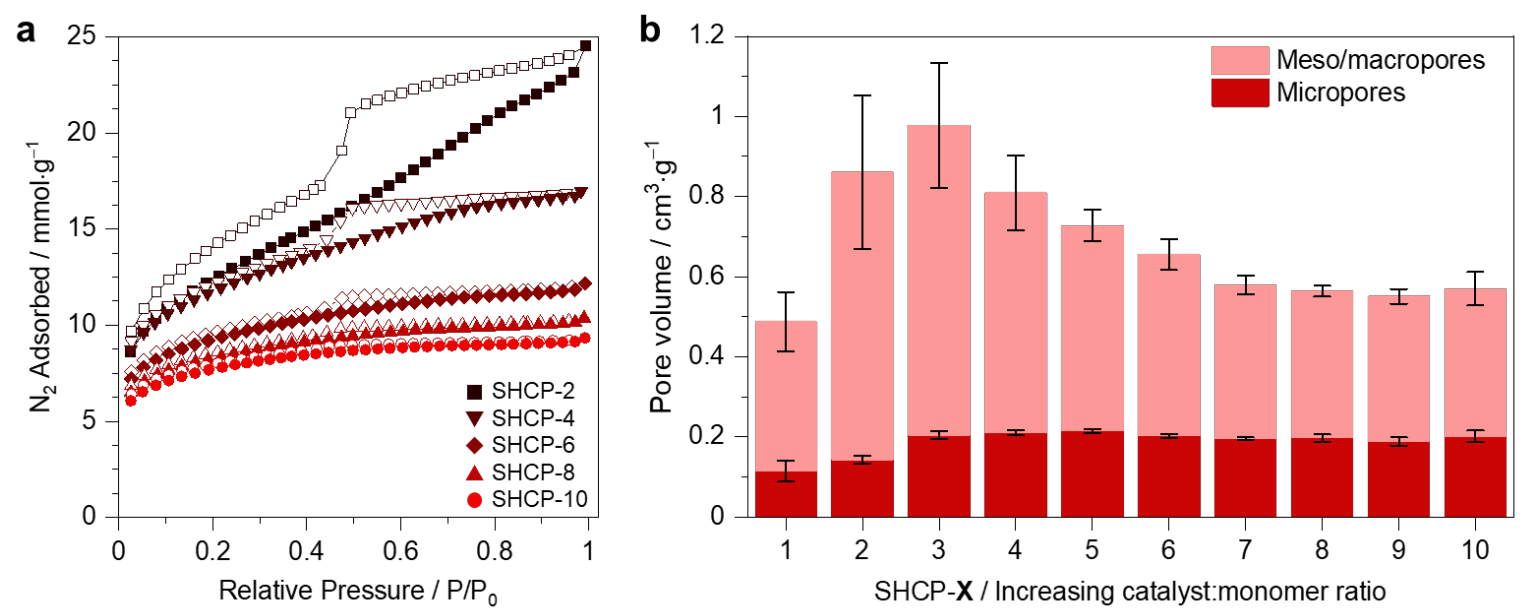

Figure 2. Textural properties of SHCPs: a) $\mathrm{N}_{2}$ adsorption-desorption isotherms for a selection of SHCPs, the remainder are shown in Figure S3. Filled symbols represent adsorption and empty symbols represent desorption. b) Pore volume of all SHCPs divided into micropore contributions (red) and mesopore/macropore contributions (pink).

The BET specific surface area (SSABET) of all SHCPs was $>530 \mathrm{~m}^{2} \cdot \mathrm{g}^{-1}$ (Table 1), with SHCP-1 displaying the lowest $S S A_{B E T}$ of $539 \mathrm{~m}^{2} \cdot \mathrm{g}^{-1}$. A trend can be seen across the catalyst set, with $\mathrm{SSA}_{\mathrm{BET}}$ increasing dramatically to a maximum of $1059 \mathrm{~m}^{2} \cdot \mathrm{g}^{-1}$ in SHCP-3 before gradually decreasing to $688 \mathrm{~m}^{2} \cdot \mathrm{g}^{-1}$ in SHCP-10. A clear trade-off between porous properties and sulfonation density was observed. In SHCP-1 and SHCP-2, inefficient hypercrosslinking occurs due to the relatively low catalyst to monomer molar ratios, as evidenced by $\mathrm{C}-\mathrm{Cl}$ in ssNMR and the presence of higher concentrations of residual Cl in XPS (Table S1). Although SHCP-3 displays the highest $S S A_{B E T}$ of the set, some evidence of remaining $\mathrm{C}-\mathrm{Cl}$ was again seen during characterisation. It is likely that the degree of sulfonation is insufficient to become significantly detrimental to the porous properties. In previous examples, the self-condensation of 4,4'bis(chloromethyl)biphenyl catalysed using triflic acid yielded HCPS with SSA $A_{B E T}$ of up to $1842 \mathrm{~m}^{2} \cdot \mathrm{g}^{-1}$, much higher than the approach herein. ${ }^{15}$ However, the production of SHCP-3 requires just one third the amount of acid catalyst used therein. When further increasing the 
chlorosulfonic acid concentration, as in SHCP-4 to SHCP-10, the resulting SSA the increased sulfonation. Even with the trade-off between SSA have excellent porous properties considering their high sulfonation density. The carbazole-based SHCP with a comparable $\mathrm{SO}_{3} \mathrm{H}$ concentration of $3.7 \mathrm{mmol} \cdot \mathrm{g}^{-1}$ displayed a $\mathrm{SSA}_{\mathrm{BET}}$ of just $346 \mathrm{~m}^{2} \cdot \mathrm{g}^{-1} \cdot{ }^{25}$

Table 1. Summary of $\mathrm{SO}_{3} \mathrm{H}$ content and textural properties of SHCPs produced from various ratios of catalyst to

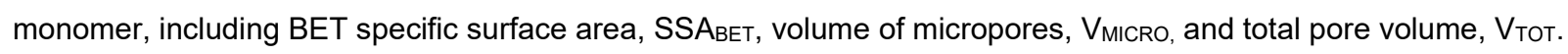

\begin{tabular}{llllll}
\hline Sample & $\begin{array}{l}\text { Catalyst:Monomer } \\
(\text { molar ratio })\end{array}$ & $\begin{array}{l}\mathrm{SO}_{3} \mathrm{H} \text { content } \\
\left(\mathrm{mmol} \cdot \mathrm{g}^{-1}\right)^{\mathrm{a}}\end{array}$ & $\mathrm{SSA}_{\mathrm{BET}}\left(\mathrm{m}^{2} \mathrm{~g}^{-1}\right)^{\mathrm{b}}$ & $\begin{array}{l}\mathrm{V}_{\text {MICRO }} \\
\left(\mathrm{cm}^{3} \cdot \mathrm{g}^{-1}\right)^{\mathrm{b}}\end{array}$ & $\mathrm{V}_{\text {TOT }}\left(\mathrm{cm}^{3} \cdot \mathrm{g}^{-1}\right)^{\mathrm{b}}$ \\
\hline SHCP-1 & 0.125 & $0.03 \pm 0.02$ & $539 \pm 123$ & $0.11 \pm 0.03$ & $0.37 \pm 0.07$ \\
SHCP-2 & 0.25 & $0.44 \pm 0.06$ & $899 \pm 165$ & $0.14 \pm 0.01$ & $0.72 \pm 0.19$ \\
SHCP-3 & 0.5 & $1.15 \pm 0.06$ & $1059 \pm 165$ & $0.20 \pm 0.01$ & $0.77 \pm 0.16$ \\
SHCP-4 & 0.75 & $1.72 \pm 0.08$ & $947 \pm 89$ & $0.21 \pm 0.01$ & $0.60 \pm 0.09$ \\
SHCP-5 & 1 & $2.20 \pm 0.13$ & $889 \pm 44$ & $0.22 \pm 0.01$ & $0.51 \pm 0.04$ \\
SHCP-6 & 1.5 & $2.82 \pm 0.09$ & $805 \pm 50$ & $0.20 \pm 0.00$ & $0.44 \pm 0.04$ \\
SHCP-7 & 2 & $3.24 \pm 0.16$ & $723 \pm 31$ & $0.20 \pm 0.00$ & $0.38 \pm 0.02$ \\
SHCP-8 & 2.5 & $3.40 \pm 0.12$ & $706 \pm 24$ & $0.20 \pm 0.01$ & $0.37 \pm 0.01$ \\
SHCP-9 & 3 & $3.60 \pm 0.17$ & $682 \pm 14$ & $0.19 \pm 0.01$ & $0.36 \pm 0.02$ \\
SHCP-10 & 4 & $3.76 \pm 0.11$ & $688 \pm 47$ & $0.20 \pm 0.01$ & $0.37 \pm 0.04$ \\
\hline
\end{tabular}

a Determined by elemental analysis

${ }^{b}$ Determined from $\mathrm{N}_{2}$ sorption isotherms at $77 \mathrm{~K}$

We analysed the thermal stability of all SHCPs using thermogravimetric analysis (TGA) in either an inert $\mathrm{N}_{2}$ atmosphere (Figure $3 \mathrm{a}$ and Figure S4) or in air (Figure S5). Negligible weight loss is observed in SHCP-1 and SHCP-2 until $>250{ }^{\circ} \mathrm{C}$. With increasing sulfonation density the weight loss prior to and during an isothermal step at $110{ }^{\circ} \mathrm{C}$ increases dramatically, reaching a maximum of $21 \mathrm{wt}$ \% for SHCP-10, indicative of a significant increase in water adsorption at high sulfonation densities. XPS measurements showed that the chemical composition of the networks remained unchanged after heating overnight at $120{ }^{\circ} \mathrm{C}$ under $\mathrm{N}_{2}$ flow, confirming degradation was not responsible for the weight loss seen in the TGA (Table S1). Finally, we employed powder X-ray diffraction (PXRD) to confirm the amorphous nature of all networks (Figure S6). 

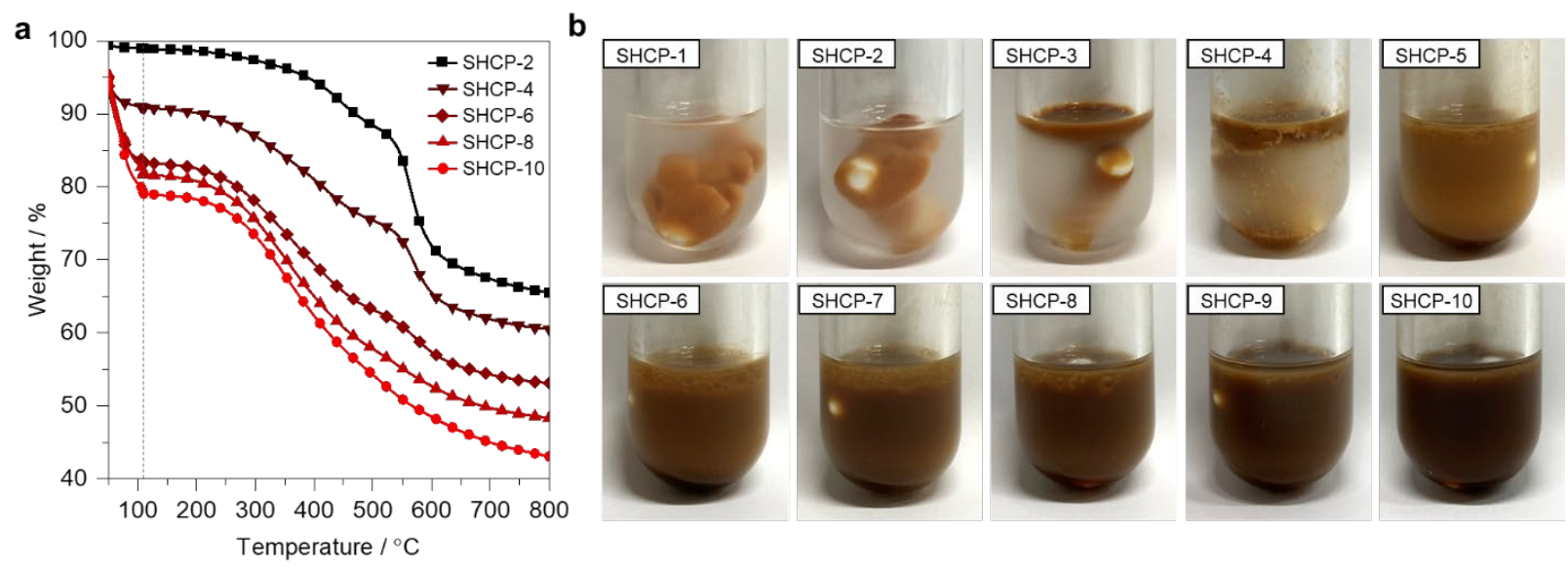

Figure 3. Varying hydrophobicity/hydrophilicity in SHCPs: a) Thermogravimetric analysis of SHCPs heated to $800{ }^{\circ} \mathrm{C}$ in a $\mathrm{N}_{2}$ atmosphere. A $10 \mathrm{~min}$ isothermal step was included at $110^{\circ} \mathrm{C}$ to remove residual water (indicated by grey dotted line). b) Photographs of all polymers in cyclohexyl acetate/cyclohexanol-water two phase systems, postcatalysis reaction.

The synthetic approach to SHCPs described herein provides a set of acidic non-soluble networks in which the sulfonation density, hydrophilicity, and textural properties can be carefully controlled. This presents a unique opportunity for the application of such a set of materials to acid-catalysed conversions in order to unveil optimal catalyst properties. To investigate the catalytic ability of SHCPs, the organic-aqueous biphasic liquid hydrolysis of cyclohexyl acetate to cyclohexanol was selected as a model reaction (reaction scheme in Table 2 and mechanism in Figure S7). We investigated substrate conversion and selectivity across our SHCP series (Table 2). Briefly, SHCP was added to a two-phase cyclohexyl acetate and water system, which was heated at $120^{\circ} \mathrm{C}$ under autogenous pressure for $3 \mathrm{~h}$. The reaction products were extracted using ethyl acetate and analysed using gas chromatography-mass spectrometry (GC-MS). A complete set of GC traces are shown in Figure S8. It is worth noting that all catalysis reactions were repeated at least four times.

Network SHCP-1 showed no measurable catalytic activity due to low acidity (S content of $\left.0.1 \mathrm{mmol} \cdot \mathrm{g}^{-1}\right)$. Control experiments with non-acidic hypercrosslinked polymer equivalents confirmed that no measurable conversion was achieved without sulfonation. The more acidic SHCP-2 (S content of $0.4 \mathrm{mmol} \cdot \mathrm{g}^{-1}$ ) gave substrate conversions of $31 \%$ with a high standard deviation of $\pm 18 \%$, suggesting that this polymer lies at a critical concentration upon which catalytic activity depends highly on network acidity. The remaining SHCPs all achieved $\geq 60 \%$ conversion of cyclohexyl acetate, outperforming both the commercial resin Amberlyst-15 and homogeneous (liquid) $\mathrm{H}_{2} \mathrm{SO}_{4}{ }^{26}$ Interestingly, high acid densities did not appear crucial for catalyst performance. 
SHCP-3 contained just $1.15 \mathrm{mmol} \cdot \mathrm{g}^{-1}$ of $\mathrm{SO}_{3} \mathrm{H}$ sites and achieved a substrate conversion of $68 \pm 8 \%$. Catalytic conversion reached a maximum of $75 \pm 2 \%$ in SHCP-5 and with increasing sulfonate content (i.e. SHCP-6 to SHCP-10), catalytic conversion decreased, reaching $60 \pm 8 \%$ in $\mathrm{SHCP}-10$, with a $\mathrm{SO}_{3} \mathrm{H}$ concentration of $3.8 \mathrm{mmol} \cdot \mathrm{g}^{-1}$. This emphasises the importance of other catalyst properties as well as acidity for this conversion. Figure $3 \mathrm{~b}$ shows each polymer in the biphasic cyclohexyl acetate/cyclohexanol and water system after catalytic conversion. Networks SHCP-5 to SHCP-8 formed quasi-stable emulsions during catalytic conversion, demonstrating amphiphilicity. As the reaction is comprised of both an organic and aqueous phase, the formation of emulsions improves the dispersion of one phase throughout the other and ensures that the catalyst resides at the organic/aqueous interface. Additionally, improved mesopore/macropore content ratios in SHCP-3 - SHCP-5 may provide good phase transfer ability, while their high surface areas ensure the substrate good access to active sites, improving conversions compared to materials with reduced mesopore/macroporosity. Selectivity in all materials was $>99 \%$ for cyclohexanol, with the formation of some trace quantities of cyclohexanone in many examples. We suspect that cyclohexanone is produced via oxidation of cyclohexanol, however as this was not confirmed it is herein considered a trace product, which was never found in quantities of $>1 \%$. The combination of amphiphilicity, high surface area, hierarchical porosity, and acidity endows SHCP-5 with the highest catalytic ability for the hydrolysis of cyclohexyl acetate to cyclohexanol. 
Table 2. Catalytic performance of SHCPs in the hydrolysis of cyclohexyl acetate to cyclohexanol. Materials are compared with sulfuric acid and the commercial resin Amberlyst-15.

\begin{tabular}{|c|c|c|}
\hline Catalyst & $\begin{array}{l}\text { Conversion } \\
(\mathrm{mol} \%)^{\mathrm{a}}\end{array}$ & $\begin{array}{l}\text { Selectivity } \\
(\mathrm{mol} \%)^{\mathrm{a}}\end{array}$ \\
\hline SHCP-1 & 0 & $>99$ \\
\hline SHCP-2 & $31 \pm 18$ & $>99$ \\
\hline SHCP-3 & $68 \pm 7$ & $>99$ \\
\hline SHCP-4 & $72 \pm 6$ & $>99$ \\
\hline SHCP-5 & $75 \pm 2$ & $>99$ \\
\hline SHCP-6 & $72 \pm 5$ & $>99$ \\
\hline SHCP-7 & $64 \pm 7$ & $>99$ \\
\hline SHCP-8 & $68 \pm 6$ & $>99$ \\
\hline SHCP-9 & $65 \pm 7$ & $>99$ \\
\hline SHCP-10 & $60 \pm 8$ & $>99$ \\
\hline $\mathrm{H}_{2} \mathrm{SO}_{4}^{\mathrm{b}}$ & 27 & 87 \\
\hline Amberlyst- $15^{\mathrm{b}}$ & 48 & 95 \\
\hline
\end{tabular}

\section{Conclusion}

We present a rapid one-pot method for the synthesis of polymeric solid acid catalysts with carefully controlled properties using a dual polymerisation catalyst and sulfonation agent. Ten SHCPs were prepared with varying porous properties, acidity, and hydrophilicity by simply varying the initial catalyst to monomer ratio. These materials could be obtained in less than $24 \mathrm{~h}$ by combining the polymerisation and sulfonation steps into a one-pot procedure, significantly increasing the speed and ease of production. This had several advantages over conventional techniques, such as eliminating the need for metal-based polymerisation catalysts, dramatically reducing the amount of toxic solvents and sulfonation agents required, and hence lowering the cost of porous heterogeneous solid-acid catalysts. Comparison with equivalent materials produced via conventional routes showed that the properties obtained in SHCPs herein are superior with respect to both acidity and surface area. The SHCPs were applied to the acidcatalysed hydrolysis of cyclohexyl acetate to cyclohexanol. Conversions of up to $75 \%$ were measured, with all networks displaying excellent selectivity (>99\%). Results strongly suggested 
that acidity is one of a number of important properties in the design of solid acid catalysts, with high acid densities even leading to reduced conversion rates. The ability to fine-tune polymer catalysts allows for the identification of key properties for further catalyst design and optimisation. We envision the application of such polymers to a plethora of acid-catalysed reactions in order to find catalyst properties most suited to each conversion, paving the way to truly tailorable low-cost acid catalysts.

\section{Notes}

The authors declare no competing financial interest.

\section{Acknowledgments}

The authors acknowledge the funding support of the University of Vienna (Austria). We thank Mag. Johannes Theiner for assistance with elemental analysis, Ing. Alexander Prado-Roller for assistance with PXRD, and Asst.-Prof. Hanspeter Kählig for assistance with ssNMR.

\section{References}

\footnotetext{
${ }^{1}$ Anastas, P. T., Warner, J. C. Green chemistry: Theory and practice. Oxford University Press: New York, 1998, 30

${ }^{2}$ Sun, L.-B., Liu, X.-Q. \& Zhou, H.-C. Design and fabrication of mesoporous heterogeneous basic catalysts. Chem. Soc. Rev., 2015, 44, 5092-5147. (DOI: 10.1039/C5CS00090D)

${ }^{3}$ Hübner, S., de Vries, J. G. \& Farina, V. Why does industry not use immobilized transition metal complexes as catalysts? Adv. Synth. Catal., 2016, 358 (1), 3-25 (DOI: 10.1002/adsc. 201500846)

${ }^{4}$ Rose, M. Nanoporous polymers: Bridging the gap between molecular and solid catalysts? ChemCatChem, 2014, $\mathbf{5}$ (6), 1166-1182 (DOI: 10.1002/cctc.201301071)

${ }^{5}$ Diercks, C. S. \& Yaghi, O. M. The atom, the molecule, and the covalent organic framework. Science, 2017,355 (6328), 923-931 (DOI: 10.1126/science.aal1585)

${ }^{6}$ Lee, J.-S. M. \& Cooper, A. I. Advances in conjugated microporous polymers. Chem. Rev., 2020, 120 (4), $2171-2214$ (DOI: 10.1021/acs.chemrev.9b00399)

${ }^{7}$ Tana, L. \& Tan, B. Hypercrosslinked porous polymer materials: Design, synthesis, and applications. Chem. Soc. Rev., 2017, 46, 3322-3356 (DOI: 10.1039/C6CS00851H)

${ }^{8}$ Li, B., Gong, R., Wang, W., Huang, X., Zhang, W., Li, H., Hu, C. \& Tan, B. A new strategy to microporous polymers: Knitting rigid aromatic building blocks by external cross-linker. Macromolecules, 2011, 44 (8), 2410-2414 (DOI: 10.1021/ma200630s)

${ }^{9}$ Wang, S. L., Zhang, C. X., Shu, Y., Jiang, S. L., Xia, Q., Chen, L. J., Jin, S. B., Hussain, I., Cooper, A. I. \& Tan, B. E. Layered microporous polymers by solvent knitting method. Sci. Adv., 2017, 3 (3), e1602610 (DOI: 10.1126/sciadv.1602610)

${ }^{10}$ Krusenbaum, A., Grätz, S., Bimmermann, S., Hutsch, S. \& Borchardt L. The mechanochemical Scholl reaction as a versatile synthesis tool for the solvent-free generation of microporous polymers. RSC Adv., 2020, 10, 25509-25516 (DOI: 10.1039/DORA05279E)

${ }^{11}$ Lee, J.-S. M., Kurihara, T. \& Horike, S. Five-minute mechanosynthesis of hypercrosslinked microporous polymers. Chem. Mater., 2020, 32 (18), 7694-7702, (DOI: 10.1021/acs.chemmater.0c01726)

${ }^{12}$ Wood, C. D., Tan, B., Trewin, A., Niu, H., Bradshaw, D., Rosseinsky, M. J., Khimyak, Y. Z., Campbell, N. L., Kirk, R., Stöckel, E. \& Cooper, A. I. Hydrogen storage in microporous hypercrosslinked organic polymer networks, Chem.

Mater., 2007, 19 (8), 2034-2048. (DOI: 10.1021/cm070356a)
} 
${ }^{13}$ Woodward, R. T. The design of hypercrosslinked polymers from benzyl ether self-condensing compounds and external crosslinkers. Chem. Commun., 2020, 56, 4938-4941. (DOI: 10.1039/D0CC01002B)

${ }^{14}$ Hou, S., Razzaquea, S. \& Tan, B. Effects of synthesis methodology on microporous organic hyper-cross-linked polymers with respect to structural porosity, gas uptake performance and fluorescence properties. Polym. Chem., 2019, 10, 1299-1311. (DOI: 10.1039/C8PY01730A)

${ }^{15}$ Schute, K., \& Rose, M. Metal-free and scalable synthesis of porous hyper-cross-linked polymers: Towards applications in liquid-phase adsorption. ChemSusChem, 2015, 8 (20), 3419-3423. (DOI: 10.1002/cssc.201500829)

${ }_{16}$ Prince, L., Guggenberger, P., Santini, E., Kleitz, F. \& Woodward, R. T. Metal-free hyper-cross-linked polymers from benzyl methyl ethers: A route to polymerization catalyst recycling. Macromolecules, 2021, 54 (19), 9217-9222. (DOI: 10.1021/acs.macromol.1c01332)

${ }_{17}$ Woodward, R. T., Stevens, L. A., Dawson, R., Vijayaraghavan, M., Hasell, T., Silverwood, I. P., Ewing, A. V., Ratvijitvech, T., Exley, J. D., Chong, S. Y., Blanc, F., Adams, D. J., Kazarian, S. G., Snape, C. E., Drage, T. C., \& Cooper, A. I. Swellable, water-and acid-tolerant polymer sponges for chemoselective carbon dioxide capture. J. Am. Chem. Soc., 2014, 136 (25), 9028-9035 (DOI: 10.1021/ja5031968)

${ }^{18}$ Rozyyev, V., Thirion, D., Ullah, R., Lee, J., Jung, M., Oh, H., Atilhan, M. \& Yavuz C. T. High-capacity methane storage in flexible alkane-linked porous aromatic network polymers. Nat. Energy, 2019, 4, 604-611 (DOI: 10.1038/s41560019-0427-x)

${ }^{19}$ Vinodh, R., Gopi, C. V. V. M., Kummara, V. G. R., Atchudan, R., Ahamad, T., Sambasivam, S., Yi, M., Obaidat, I. M. \& Kim, H.-J. A review on porous carbon electrode material derived from hypercross-linked polymers for supercapacitor applications. J. Energy Storage, 2020, 32, 101831. (DOI: 10.1016/j.est.2020.101831)

${ }^{20} \mathrm{Gu}, \mathrm{Y}$., Son, S. U., Li, T. \& Tan, B. Low-cost hypercrosslinked polymers by direct knitting strategy for catalytic applications. Adv. Funct. Mater., 2021, 31, 12, 2008265 (DOI: 10.1002/adfm.202008265)

${ }^{21}$ Dong, K., Zhang, J., Luo, W., Su, L. \& Huang, Z. Catalytic conversion of carbohydrates into 5-hydroxymethyl furfural over sulfonated hyper-cross-linked polymer in DMSO. Chem. Eng. J., 2018, 334, 1055-1064 (DOI: 10.1016/j.cej.2017.10.092)

${ }^{22}$ Zhang, J., Dong, K., Luo, W. \& Guan, H. Catalytic upgrading of carbohydrates into 5-ethoxymethylfurfural using SO3H functionalized hyper-cross-linked polymer based carbonaceous materials. Fuel, 2018, 234, 664-673 (DOI: 10.1016/j.fuel.2018.07.060)

${ }^{23}$ Schukraft, G. E. M., Woodward, R. T., Kumar, S., Sachs, M., Eslava, S. \& Petit C. Hypercrosslinked polymers as a photocatalytic platform for visible-light-driven $\mathrm{CO}_{2}$ photoreduction using $\mathrm{H}_{2} \mathrm{O}$. ChemSusChem, 2021, 14, 1720-1727. (DOI: 10.1002/cssc.202002824)

${ }^{24}$ Wang, S., Xu, M., Peng, T., Zhang, C., Li, T., Hussain, I., Wang, J. \& Tan, B. Porous hypercrosslinked polymer-TiO $2^{-}$ graphene composite photocatalysts for visible-light-driven $\mathrm{CO}_{2}$ conversion. Nat. Commun., 2019, 10, 676. (DOI: 10.1038/s41467-019-08651-x)

${ }^{25}$ Bhunia, S., Banerjee, B. \& Bhaumik, A. A new hypercrosslinked supermicroporous polymer, with scope for sulfonation, and its catalytic potential for the efficient synthesis of biodiesel at room temperature. Chem. Commun., 2015, 51, 5020-5023 (DOI: 10.1039/C4CC09872B)

${ }^{26}$ Lei, Y., Zhang, M., Leng, G., Ding, C. \& Ni Y. $\mathrm{SO}_{3} \mathrm{H}$-functionalized porous organic polymer with amphiphilic and swelling properties: A highly efficient solid acid catalyst for organic transformations in water. Microporous Mesoporous Mater. 2020, 299, 110110. (DOI: 10.1016/j.micromeso.2020.110110)

${ }^{27}$ Stephenson, A., Li, B., Chen, L., Clowes, R., Briggs, M. E. \& Cooper A. I. Efficient separation of propane and propene by a hypercrosslinked polymer doped with $\mathrm{Ag}(\mathrm{I})$. J. Mater. Chem. A, 2019, 7, 25521-25525 (DOI: 10.1039/C9TA07510K)

${ }^{28}$ Xiong, Y., Woodward, R. T., Danaci, D., Evans, A., Tian, T., Azzan, H., Ardakani, M. \& Petit, C. Understanding tradeoffs in adsorption capacity, selectivity and kinetics for propylene/propane separation using composites of activated carbon and hypercrosslinked polymer. Chem. Eng. J., 2021, 426, 131628 (DOI: 10.1016/j.cej.2021.131628)

${ }^{29}$ James, A. M., Harding, S., Robshaw, T., Bramall, N., Ogden, M. D. \& Dawson R. Selective environmental remediation of strontium and cesium using sulfonated hyper-cross-linked polymers (SHCPs). ACS Appl. Mater. Interfaces, 2019, 11 (25), 22464-22473 (DOI: 10.1021/acsami.9b06295)

${ }^{30}$ Xiong, G., Zhang, Q., Ren, B., You, L., Ding, F., He, Y., Fan, X., Wang, N. \& Sun, Y. Highly efficient and selective adsorption of cationic dyes in aqueous media on microporous hyper crosslinked polymer with abundant and evenly dispersed sulfonic groups. ChemistrySelect, 2020, 5, 6541 -6546 (DOI: 10.1002/slct.202000927) 
${ }^{31}$ Matsuda, M., Funabashi, K., Yusa, H. \& Kikuchi, M. Influence of functional sulfonic acid group on pyrolysis characteristics for cation exchange resin. J. Nucl. Sci. Technol., 1987, 24 (2), 124-128 (DOI:

10.1080/18811248.1987.9735785)

32 Thommes, M., Kaneko, K., Neimark, A. V., Olivier, J. P., Rodriguez-Reinoso, F., Rouquerol, J. \& Sing, K. S. W. Physisorption of gases, with special reference to the evaluation of surface area and pore size distribution (IUPAC Technical Report). Pure Appl. Chem. 2015, 87, 1051-1069. (DOI: 10.1515/pac-2014-1117)

${ }^{33}$ Bilodeau, S., Florek, J. \& Kleitz, F. Reassessing the physicochemical properties of ordered mesoporous polymer and copolymer nanocasts. Chem. Ing. Tech., 2021, 93 (6), 916-928. (DOI: 10.1002/cite.202000238) 